\title{
Acute eosinophilic pneumonia associated with 2009 influenza $A$ (H1N1)
}

\author{
Eun Ju Jeon, ${ }^{1}$ Kwang Hyun Kim, ${ }^{2}$ Kyung Hoon $\mathrm{Min}^{2}$
}

'Department of Internal Medicine, Chung-Ang University College of Medicine, Seoul, South Korea ${ }^{2}$ Department of Internal Medicine, Armed Forces Capital Hospital, Seongnam, South Korea

\section{Correspondence to} Dr Kyung Hoon Min, Department of Internal Medicine, Armed Forces Capital Hospital, Yuldong, Bundang-gu, Seongnam, 463-040, South Korea; mkhmd@jbnu.ac.kr

Received 9 December 2009 Accepted 15 December 2009

\section{ABSTRACT}

Acute eosinophilic pneumonia (AEP) is a cause of acute respiratory failure, and differs from other eosinophilic pneumonias by its acute onset, presence of fever and acute hypoxaemia. Cases of 2009 influenza A (H1N1) virus infection have included rapidly progressive lower respiratory tract disease resulting in respiratory failure. We describe the case of a young patient who showed dyspnoea, fever and rapid radiological progression and was simultaneously diagnosed with AEP and 2009 influenza $A$ (H1N1) infection, which was diagnosed by using bronchoalveolar lavage (BAL). We suggest that 2009 influenza A (H1N1) virus should be considered as a cause of AEP, and BAL can provide further information, especially in patients with severe lower respiratory disease.

A 20-year-old man was admitted to a nearby hospital with symptoms including fever $\left(38.3^{\circ} \mathrm{C}\right)$, dyspnoea and chest discomfort for 2 days. He had no history of smoking, drug use or exposure to dust and disease, such as allergies or HIV infection. Chest auscultation revealed inspiratory crackles in both lower lung fields. A chest radiograph and CT showed ill-defined ground-glass opacities with interlobular septal thickening and minimal bilateral pleural effusion (figure 1A,B). The patient's arterial blood gas analysis was pH 7.46, $\mathrm{PaO}_{2} 70 \mathrm{~mm} \mathrm{Hg}, \mathrm{PaCO}_{2}$ $36.2 \mathrm{~mm} \mathrm{Hg}$ and $95 \% \mathrm{O}_{2}$ saturation. The results of other laboratory tests revealed white blood cells $13.0 \times 10 \% / 1$ (neutrophils $73 \%$, lymphocytes $14.2 \%$, monocytes $8.6 \%$ and eosinophils $4.2 \%$ ) and Creactive protein $7.2 \mathrm{mg} / \mathrm{dl}$. The clinician prescribed oseltamivir (75 mg twice daily) and obtained a throat specimen for real-time reverse transcriptase-PCR (RT-PCR) test for 2009 influenza A (H1N1). On the day following admission, a chest radiograph showed further aggravation (figure 1C). The patient was transferred to our hospital and admitted to an isolation room in the intensive care unit. We performed bedside bronchoalveolar lavage (BAL) that recovered $0.36 \times 10^{9}$ cells $/ 1$ with a differential of eosinophils 39\%, lymphocytes $29 \%$ and polymorphonuclear leucocytes $5 \%$ (figure 2 ). We requested an RT-PCR test for 2009 influenza A (H1N1) using the specimen obtained through BAL. Cultures of blood, urine, sputum and BAL specimens did not show growth of any pathogenic organisms. We tested for allergies (serum total immunoglobulin (Ig) E, MAST allergen inhalant), parasite infection (serum parasite-specific antibody IgG), and conducted an HIV-1 enzyme immunoassay screening test; all the results were within the normal range. On the basis of the acute eosinophilic pneumonia (AEP) criteria, we diagnosed this case as
AEP, and prescribed prednisone (30 $\mathrm{mg}$ twice daily) in addition to oseltamivir. The 2009 influenza A (H1N1) RT-PCR results were reported on hospital day 2. The test on the upper respiratory tract specimen (throat swab) was negative, but the test for the lower respiratory tract specimen (BAL) was positive. Consequently, 2009 influenza A (H1N1) infection was diagnosed. The patient took oseltamivir (75 mg twice daily) for 5 days and prednisone (30 mg twice daily) for 7 days; prednisone was then tapered off for 7 days. After the patient started taking prednisone, his symptoms improved immediately and fever subsided. A chest radiograph showed complete resolution of the pulmonary lesion (figure 1D).

\section{DISCUSSION}

AEP is an acute febrile respiratory illness with severe hypoxaemia, diffuse pulmonary infiltrates and an increase in BAL eosinophils. One study has suggested that AEP is an acute hypersensitivity reaction to an unidentified inhaled antigen. ${ }^{1}$ The following observations are consistent with this hypothesis. Onset of cigarette smoking, alterations in smoking habits, unusual outdoor activities and inhalation of cocaine or heroin are the known reported causes. ${ }^{2-6}$ There is also evidence that some infections can cause AEP. In patients who received lung allografts, AEP was attributed to Aspergillus species, coxsackie A2 virus and Pseudomonas maltophilia. ${ }^{7}$ In the present case, we tried to exclude known possible causes of AEP. The patient had no history of allergic disease, smoking, drug use or exposure to dust. The tests for allergies, parasite infection, HIV infection and bacterial infection were all negative, and there was no peripheral eosinophilia. Consequently, the only one possible cause of AEP was 2009 influenza A (H1N1).

2009 influenza A (H1N1) virus has emerged to cause the first pandemic of the 21st century, and can cause acute respiratory distress syndrome in previously healthy persons. ${ }^{8}$ In most cases, 2009 influenza A (H1N1) virus is confirmed by using an RT-PCR test that is performed on a swab obtained from the upper respiratory tract. The performance of RT-PCR assays specific for 2009 influenza A (H1N1) has not been established for BAL and tracheal aspirates. However, the US Centers for Disease Control and Prevention recommends that lower respiratory tract specimens should be collected and tested to improve diagnostic yield in patients with severe lower respiratory disease (eg, suspected viral pneumonia) who are intubated or undergoing bronchoscopy. ${ }^{9}$ One report has demonstrated that investigators assayed 21 patients who 
Figure 1 (A) Chest radiograph on admission showed mixed reticular and ground-glass opacity on both mid and lower lung fields with bilateral blunting of the costophrenic angles. (B) Chest CT showed ill-defined ground-glass opacities with interlobular septal thickening and minimal bilateral pleural effusion. (C) Chest radiograph on 1 day after admission showed further aggravation of the pulmonary lesion. (D) Chest radiograph on the day of discharge showed complete resolution of the pulmonary lesion.
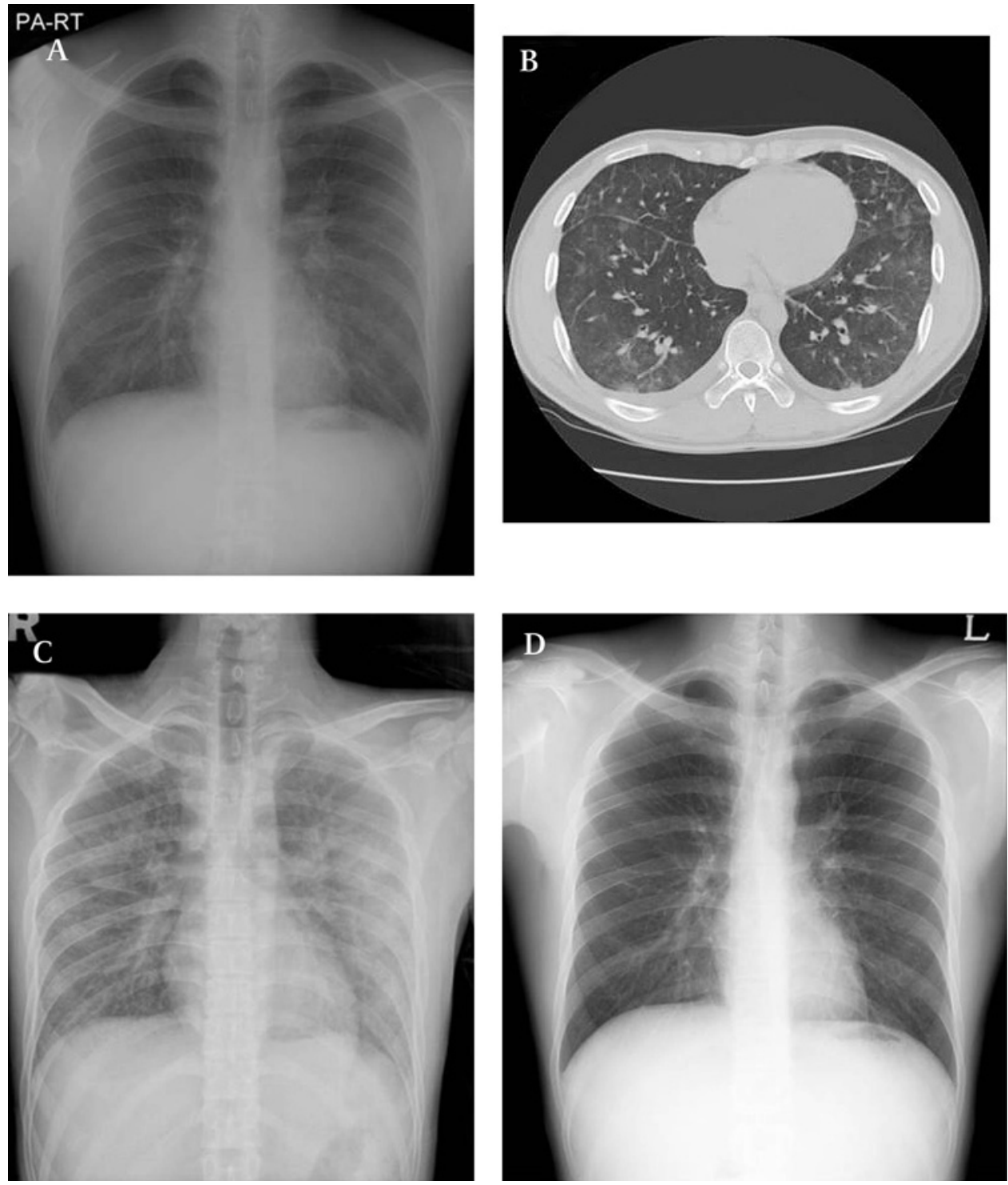

were confirmed as having 2009 influenza A (H1N1) infection. Investigators obtained upper respiratory tract specimens from the nose or throat, and lower respiratory tract specimens using bronchoscopy. Specimens from the lower respiratory tract were positive for 2009 influenza A (H1N1) in all patients when tested with RT-PCR, but specimens from the upper respiratory tract were positive in only 17 of 21 patients (81\%). ${ }^{10}$ In accordance with this previous study, our present case showed that RT-PCR was only positive in the lower respiratory tract specimen.
Although the patient in this report received oseltamivir, if a lower respiratory tract specimen had not been obtained we could not have confirmed the cause of acute febrile lower respiratory disease.

To our knowledge, this is the first report of AEP associated with 2009 influenza A (H1N1). We conclude that 2009 influenza A (H1N1) virus should be considered as a cause of AEP. Although BAL is not the essential specimen for study in the diagnosis of all patients presenting with suspected 2009 influenza A (H1N1), it
Figure 2 Centrifuged preparation of the bronchoalveolar lavage (BAL) fluid. Eosinophils accounted for $39 \%$ of the total cells (Diff-Quik stain, $A: \times 200, B$ : $\times 400)$.
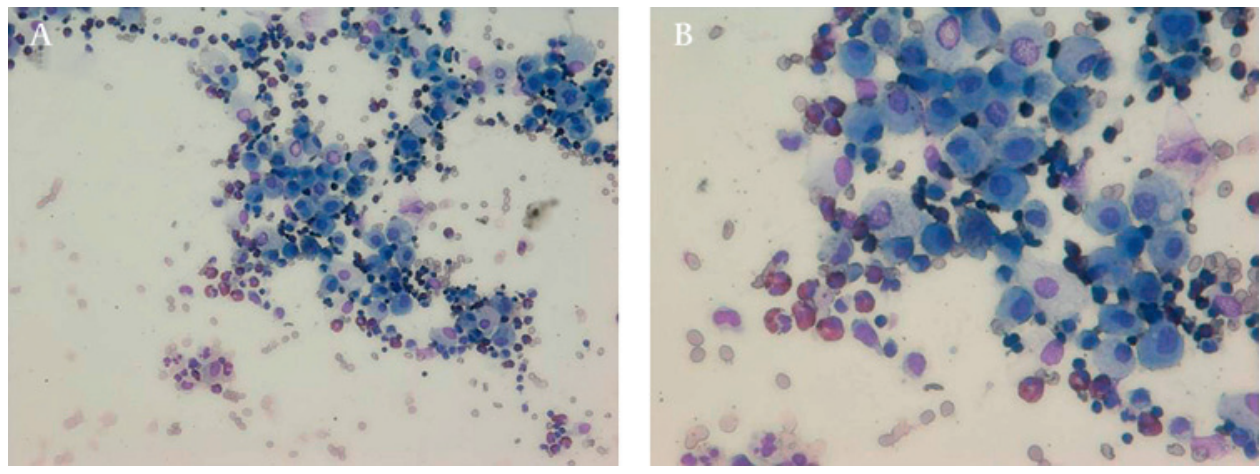
can provide further information for clinicians, especially in patients with severe lower respiratory disease.

Competing interests None.

Patient consent Obtained.

Provenance and peer review Not commissioned; externally peer reviewed.

\section{REFERENCES}

1. Badesch DB, King TE Jr, Schwarz Ml. Acute eosinophilic pneumonia: a hypersensitivity phenomenon?. Am Rev Respir Dis 1989;139:249-52.

2. Miki K, Miki M, Nakamura Y, et al. Early-phase neutrophilia in cigarette smokeinduced acute eosinophilic pneumonia. Intern Med 2003;42:839-45.

3. Uchiyama H, Suda T, Nakamura Y, et al. Alterations in smoking habits are associated with acute eosinophilic pneumonia. Chest 2008;133:1174-80.
4. Rom WN, Weiden M, Garcia R, et al. Acute eosinophilic pneumonia in a New York city firefighter exposed to World Trade Center dust. Am J Respir Crit Care Med 2002; 166:797-800

5. McCormick M, Nelson T. Cocaine-induced fatal acute eosinophilic pneumonia: a case report. WMJ 2007;106:92-5.

6. Brander PE, Tukiainen P. Acute eosinophilic pneumonia in a heroin smoker. Eur Respir J 1993;6:750-2.

7. Yousem SA. Graft eosinophilia in lung transplantation. Hum Pathol 1992;23:1172-7

8. Perez-Padilla R, de la Rosa-Zamboni D, Ponce de Leon S, et al. Pneumonia and respiratory failure from swine-origin influenza a (H1N1) in Mexico. N Engl J Med 2009;361:680-9.

9. United States Centers for Disease Control and Prevention. Interim recommendations for clinical use of influenza diagnostic tests during the 2009-10 influenza season. www.cdc.gov/h1n1flu/guidance/diagnostic_tests.htm (accessed 9 Dec 2009).

10. Blyth CC, Iredell JR, Dwyer DE. Rapid-test sensitivity for novel swine-origin influenza a (H1N1) virus in humans. N Engl J Med 2009;361:2493.

\section{Pulmonary puzzle}

\section{ANSWER}

From the question on page 214

Churg-Strauss syndrome (CSS) was diagnosed on the basis of extensive vasculitis in a sural nerve biopsy, tissue eosinophilia in a cecum biopsy (see figure in online supplement) and peripheral eosinophilia (11\%, observed only once). ${ }^{1}$ The cecum biopsy was performed because anaemia had become microcytic; pathological analysis was consistent with an eosinophilic gastroenteritis. p-Antineutrophil cytoplasmic (titre 1:1280) and anti-myeloperoxidase $(657 \mathrm{U} / \mathrm{ml})$ antibodies were strongly positive. CSS disease activity markers soluble interleukin-2 receptor $(7748$ pg/ $\mathrm{ml})$ and eosinophilic cationic protein $(13.2 \mathrm{mg} / \mathrm{l})$ were elevated. ${ }^{2}$ There was no history of the use of antileukotriene agents or acetaminophen. Although no single CT image is pathognomonic for CSS, ground-glass attenuation appears to be the most consistent feature. ${ }^{3}$ Pulmonary fibrosis is not usually mentioned as a complication of $\mathrm{CSS}^{1}$ but has been reported previously ${ }^{4}$ and may represent the final common pathway of untreated disease.

The following points can be learnt from this case. CSS can present with symptoms resembling rheumatoid arthritis ${ }^{5}$ but joint involvement is not usually mentioned as a manifestation of CSS. ${ }^{1}$ CSS may be present without a formal diagnosis of asthma because pulmonary vascular disease can also be characterised by low diffusing capacity without obstruction. ${ }^{6}$ Limited testing for polyneuropathy in the context of diabetes mellitus is usually recommended. ${ }^{7}$ However, severe pain, asymmetry and predominant or progressive motor symptoms (eventually all present in this patient) should serve as red flags and warrant further testing. ${ }^{7}$ Early recognition of CSS is important because treatment may stop the potentially dangerous progression of disease and reverse symptoms. In that regard, the presence of allergic rhinitis should have served as a diagnostic clue. Continuous low- dose steroid treatment may have disguised other diagnostic clues such as eosinophilia in blood and lavage fluid. Instead, a bronchoscopic or surgical lung biopsy early in the course may have expedited diagnosis. This case vividly demonstrates one of medicine's challenges - the necessity to search for all possible diagnoses to avoid missing one, but also to avoid missing a single unifying diagnosis during this search. This case also illustrates the pitfalls of subspecialisation. The rheumatologist, respiratory physician and neurologist found alternative explanations for symptoms of CSS within their areas of expertise, obscuring the larger picture.

The patient was treated with prednisone (30 mg daily) and azathioprine (100 mg daily). Fever did not return, polyneuropathy improved and erythrocyte sedimentation rate, C-reactive protein and haemoglobin normalised.

Supplementary figure is published online only at http://thx.bmj.com/content/vol65/ issue3.

Thorax 2010:65:270. doi:10.1136/thx.2009.128397a

\section{REFERENCES}

1. Noth I, Strek ME, Leff AR. Churg-Strauss syndrome. Lancet 2003;361:587-94.

2. Schmitt WH, Csernok E, Kobayashi S, et al. Churg-Strauss syndrome: serum markers of lymphocyte activation and endothelial damage. Arthritis Rheum 1998;41:445-52.

3. Worthy SA, Müller NL, Hansell DM, et al. Churg-Strauss syndrome: the spectrum of pulmonary CT findings in 17 patients. AJR Am J Roentgenol 1998;170:297-300.

4. Ando Y, Okada F, Matsumoto S, et al. Thoracic manifestation of myeloperoxidaseantineutrophil cytoplasmic antibody (MPO-ANCA)-related disease. CT findings in 51 patients. J Comput Assist Tomogr 2004;28:710-16.

5. Diri E, Buscemi DM, Nugent KM. Churg-Strauss syndrome: diagnostic difficulties and pathogenesis. Am J Med Sci 2003;325:101-5.

6. Kim YK, Lee KS, Chung MP, et al. Pulmonary involvement in Churg-Strauss syndrome: an analysis of CT, clinical, and pathologic findings. Eur Radiol 2007:17:3157-65.

7. England JD, Asbury AK. Peripheral neuropathy. Lancet 2004;363:2151-61. 